\title{
Ficha Antropométrica no Núcleo de Apoio a Saúde da Família: o que medir e para que medir?
}

\author{
Anthropometric record cards used at Family Health Support \\ Centers: What should be measured and why?
}

\author{
Marcius de Almeida Gomes 1,2 \\ Carmem Cristina Beck \\ Maria de Fátima da Silva Duarte ${ }^{3}$ \\ Edio Luiz Petroski ${ }^{3}$
}

\begin{abstract}
1 Universidade do Estado da Bahia. Departamento de Educação-Campus XII Salvador, Bahia. Brasil.
\end{abstract}

2 Doutorando(a) do Programa de Pós-graduação em Educação Física, Universidade Federal de Santa Catarina, Centro de Desportos. Florianópolis, SC. Brasil

3 Universidade Federal de Santa Catarina.Programa de Pós-graduação em Educação Física. Centro de Desportos, Bolsista $\mathrm{PQ} / \mathrm{CNPQ}$. Florianópolis, SC. Brasil

Recebido em 01/08/08 Revisado em 17/11/08 Aprovado em 05/12/08
Resumo - A aplicação da antropometria representa um importante instrumento para a efetivação de práticas na saúde pública. Diante da inserção do professor de educação física no Núcleo de Apoio à Saúde da Família, torna-se necessária a apropriação de métodos e técnicas antropométricas, a fim de ampliar a sua contribuição em iniciativas na área da saúde. Para tanto, este estudo tem como objetivo sugerir uma proposta de aplicação da antropometria no Núcleo de Apoio à Saúde da Família, apontando procedimentos para o ambiente da saúde pública e considerando os princípios propostos para a promoção à saúde no Brasil. Além de proporcionar a detecção de fatores de risco à saúde, a aplicação da antropometria deve evidenciar as estratégias de promoção à saúde, possibilitando que os profissionais envolvidos possam mediar às ações de controle e de prevenção por meio da prática efetiva da educação à saúde.

Palavras-chave: Antropometria; Atividade Física; Atenção Primária; Programa Saúde da Família.

Abstract - Anthropometry is an important instrument for implementing professional practices in the public health care. In view of the participation of physical education teachers at Family Health Support Centers, the use of anthropometric methods becomes necessary in order to increase its contribution to health-care initiatives. Therefore, this study aims at proposing the application of anthropometry at Family Health Support Centers, indicating procedures for the public health-care environment and considering the principles used for health promotion in Brazil. Besides detecting health risk factors, the application of anthropometry should highlight health-care promotion strategies, so that it allows the involved professionals to mediate control and preventive measures through effective health-care education.

Key words: Anthropometry; Physical Activity; Primary Care; Family Health Programs. 


\section{INTRODUÇÃO}

Nas últimas décadas a utilização da antropometria na saúde pública tem contribuído substancialmente para a identificação de grupos com problemas nutricionais e com fatores de risco associados às doenças, bem como na elaboração de iniciativas para a vigilância nutricional.

Considerando a proposta de promoção à saúde $^{1}$ e a importância da estratégia global, conhecida como "Saúde para todos no ano 2000", na segunda metade da década de 90 , a política de saúde no Brasil, apresentou uma significativa mudança, onde a Atenção Básica passou a ser área de concentração de esforços, programas e investimentos ${ }^{3}$.

Com a criação do Programa Saúde da Família, a partir de 1994, evidenciaram-se as atividades de promoção à saúde e de prevenção de doenças, com ações educativas numa perspectiva capaz de orientar as diferentes práticas no campo da saúde 4 .

Porém, percebe-se que a utilização da antropometria na saúde pública tem se restringido a diagnósticos populacionais ${ }^{5}$, limitando sua aplicação para o âmbito da promoção a saúde.

Diante da inserção do professor de Educação Física nas estratégias do Sistema Único de Saúde (SUS), por meio dos Núcleos de Apoio a Saúde da Família $^{1}(\mathrm{NASF})^{6}$ torna-se necessária à apropriação dos métodos e técnicas antropométricas, a fim de ampliar a contribuição por parte destes profissionais em iniciativas na área da saúde pública.

Para tanto, este trabalho tem como objetivo sugerir uma proposta de aplicação da antropometria no NASF, apontando procedimentos para o contexto da saúde pública e considerando os princípios e as estratégias utilizadas para a prática da promoção à saúde no Brasil.

\section{PROPOSIÇÃO E LOGÍSTICA DA ANTROPOMETRIA PARA O NASF}

O impacto negativo de determinados agravos não transmissíveis (obesidade, hipertensão e diabetes, etc) para a saúde pública, fez com que o Ministério da Saúde desde a década de 70, implantasse o Sistema de Vigilância Alimentar e Nutricional ${ }^{5}$ (SISVAN).

\footnotetext{
1 Art. $1^{\circ}$ da Portaria Ministerial de Saúde $\mathrm{n}^{\circ} 154$, de 24 de janeiro de 2008, resolve criar os NASF, com o objetivo de ampliar a abrangência e o escopo das ações da atenção básica, bem como sua resolubilidade, apoiando a inserção da Estratégia de Saúde da Família na rede de serviços e o processo de territorialização e regionalização a partir da atenção básica.
}

Atualmente, como parte da Política Nacional de Alimentação e Nutrição, o SISVAN monitoriza as condições dos grupos menos favorecidos da população, utilizando métodos de avaliação rápida e permanente, de fatores que influenciam os padrões de consumo alimentar e de estado nutricional.

Nota-se, que dentre as diferentes etapas da avaliação do estado nutricional no SISVAN, a avaliação antropométrica é utilizada como importante ferramenta, uma vez que as condições nutricionais podem ser expressas por medidas corporais.

Porém, a Secretaria de Atenção à Saúde ${ }^{7}$ reconhece que os serviços de saúde precisam se sensibilizar para a importância das medidas antropométricas, como forma de garantir confiabilidade e qualidade no cuidado à saúde de crianças, adolescentes, adultos e idosos.

Pensando na ampliação e na utilização das informações fornecidas pela antropometria, com novas medidas e indicadores, é que se propõe a implantação da ficha antropométrica para o NASF.

O NASF surge com o intuito de agregar outros profissionais de saúde, os quais não fazem parte da equipe mínima da Estratégia de Saúde da Família (ESF), composta por um médico de família, um enfermeiro, um auxiliar de enfermagem e seis agentes comunitários de saúde e que, quando ampliada, conta ainda com: um dentista, um auxiliar de consultório dentário e um técnico em higiene dental ${ }^{8}$.

No desenvolvimento de suas atividades, a equipe de saúde da família, de característica multiprofissional, deve: conhecer as famílias do território de abrangência; identificar os problemas de saúde e as situações de riscos existentes na comunidade; elaborar um plano e uma programação de atividades para enfrentar os determinantes do processo saúde/doença; desenvolver ações educativas e intersetoriais relacionadas aos problemas de saúde identificados; e prestar assistência integral às famílias sob sua responsabilidade no âmbito da Atenção Básica?.

Um dos propósitos do NASF é potencializar as ações de prevenção e promoção à saúde na atenção primária, obedecendo às demandas das áreas de abrangências das equipes de saúde da família ${ }^{6}$.

Como caráter estratégico, a atuação do professor de Educação Física no NASF, deve buscar a inclusão de toda a comunidade adstrita, evitando a restrição de suas ações apenas às populações já adoecidas ou mais vulneráveis ${ }^{6}$.

Gomes ${ }^{10}$ reconhece que a Estratégia de Saúde da Família (ESF) apresenta importantes mecanismos (a atuação multidisciplinar, a visita domiciliar, 
o controle por área de abrangência e a formação dos grupos focais) que resultam em contribuições expressivas para a promoção à saúde e prevenção de doenças. Diante da dinâmica e aplicação destes mecanismos, a utilização da ficha antropométrica pode servir como um importante instrumento para o processo de territorialização nas áreas de abrangências onde irá atuar o professor de educação física.

Logo, aproveitando este contexto da ESF e o detalhamento das ações para o professor de educação física propostas pelo NASF, será apresentada a seguir a logística dos procedimentos para a aferição das medidas antropométricas, destacando: quem e como realizar, onde realizar e os equipamentos utilizados.

\section{LOGÍSTICA}

A aplicação da ficha antropométrica no NASF acontecerá em dois momentos: 1) com medidas de triagem; e 2) com medidas complementares.

As medidas de triagem devem ser realizadas pela Agente Comunitário de Saúde (ACS), nos domicílios adstritos à sua respectiva micro área, o qual estará munido de um estadiômetro e de uma balança portáteis, e de uma fita antropométrica, para realizar as medidas de massa corporal, estatura e o perímetro de cintura (PC).

Após a realização das medidas (massa corporal, estatura e PC), a ACS classifica o risco associado ao excesso de peso corporal e a distribuição de gordura, por meio do cálculo do índice de massa corporal (IMC) e do valor do PC.

Os indivíduos que não apresentarem risco serão convidados a participar de atividades de educação em saúde, a fim de reforçar a importância do comportamento saudável, destacando as possibilidades de aderência (autonomia). Já, os indivíduos que apresentarem risco aumentado, serão encaminhados para a realização das medidas complementares na Unidade Básica de Saúde (UBS), sendo previamente agendadas e registradas em um cartão antropométrico por ela ACS.

Na UBS, antes da realização das medidas complementares, o professor de educação física informará aos indivíduos os objetivos, os procedimentos de medidas e as possíveis recomendações. As medidas complementares, dobras cutâneas e perímetros, serão aferidas conforme a indicação do protocolo de medidas, a fim de identificar outros componentes da composição corporal (\%G e massa magra).

O professor de educação física deverá contar com um adipômetro científico, uma fita antropo- métrica e um lápis dermográfico para realização das medidas.

A aplicação em duas etapas (triagem e complementar) se justifica pela necessidade de uma intervenção mais direcionada àqueles que apresentam riscos associados às doenças nutricionais, metabólicas ou cardiovasculares, a fim de receber orientação para adoção de comportamentos saudáveis, e refletir sobre as condições econômicas, ambientais e sociais (barreiras) que interferem na sua mudança de comportamento.

A participação em grupos focais, com palestras, seminários/círculo de cultura ${ }^{11}$ e em atividades pontuais (Ex: semana de avaliação e orientação de atividades físicas e saúde) deve ser algo constante nesta iniciativa, promovendo práticas de educação a saúde que estimulem a utilização dos espaços comunitários, sejam estes públicos ou privados, presentes na área de abrangência.

Para exemplificar, a Figura 1 apresenta o modelo de cobertura e distribuição da estrutura da ESF e a abrangência do NASF. Este exemplo permite desenvolver iniciativas especificas para cada uma das 16 micros áreas ou para cada uma das quatro áreas de abrangência, identificando as medidas antropométricas e outras variáveis (barreiras e estágios de comportamento) para atividade física, percepção do ambiente e estilo de vida ${ }^{10}$. Este diagnóstico facilita a construção e a elaboração de políticas públicas para o controle e prevenção dos agravos específicos, a exemplo das DANTS, identificados pela cobertura do NASF.

Abrangência do Núcleo de Apoio a Saúde da Família

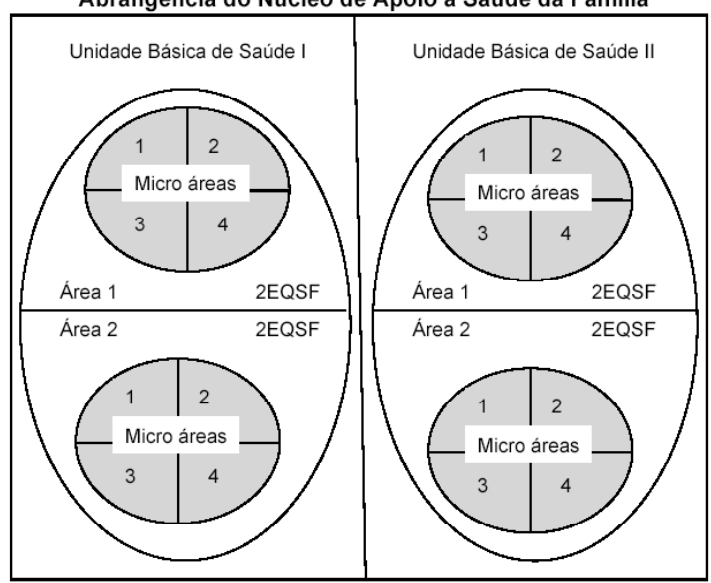

Figura 1. Modelo da cobertura para o NASF, com quatro áreas de abrangência dividida em quatro micro áreas cada, oito equipes de saúde da família (EQSF), duas unidades básicas de saúde, com atendimento de mil famílias por equipe.

A aplicação da antropometria no NASF se dará com os seguintes objetivos: 
- Realizar triagem das comunidades adstritas por meio dos indicadores de risco associados ao baixo peso, a obesidade e as doenças plurimetabólicas;

- Caracterizar as áreas e micro áreas de abrangência das Equipes de Saúde da Família segundo os indicadores antropométricos associadas aos fatores de risco predisponentes às doenças;

- Monitorar as mudanças na composição corporal, associadas aos distúrbios metabólicos e endócrinos, e às alterações morfológicas relacionadas ao processo de envelhecimento humano com destaque para a perda da massa corporal magra (MCM);

- Elaborar e desenvolver intervenções e programas nos campos da educação física e da nutrição para a prevenção de doenças e para a promoção à saúde em níveis de comunidade e individual;

- Supervisionar e controlar os efeitos de intervenções e tratamentos propostos; e,

- Identificar e desenvolver temas geradores², a fim de estimular a reflexão sobre as mudanças da composição corporal e a influência de outros aspectos (sociais, culturais, econômicos e comportamentais);

A proposta aqui apresentada atenta para a efetivação da prática da educação à saúde, aproximando o conteúdo da antropometria ao senso comum, independente do diagnóstico de alguma situação de risco ou não. Informações como estas são importantes para aumentar o controle sobre a qualidade de vida e saúde, contribuindo para a construção do empowerment ${ }^{1}$.

Como uma das estratégias da promoção à saúde o empoderamento (empowerment) é definido como a participação popular na direção dos assuntos de saúde, bem como o acesso total e contínuo às informações e às oportunidades de aprendizagem nesta área, ou seja, a aquisição de poder técnico e consciência política para atuar em prol de sua saúde ${ }^{12}$.

O modelo apresentado neste trabalho (Quadro 2) sugere os seguintes componentes e procedimentos de medida:

Identificação: com dados pessoais (nome, sexo e idade) e referências da UBS (bairro, $\mathrm{n}^{\circ}$ da área, $\mathrm{n}^{\circ}$ da micro área e identificação da ACS);

Dados antropométricos: com medidas de massa

2 O tema gerador é entendido como sendo o assunto que centraliza o processo da educação, sendo sua escolha fruto de uma mediação entre as responsabilidades dos educadores (profissionais de saúde) e os alunos (indivíduos) refletindo e dialogando sobre o entendimento do problema ${ }^{11}$. corporal $(\mathrm{kg})$ e estatura $(\mathrm{m})$ segundo Alvarez e Pa$\operatorname{van}^{13}$, perímetros $(\mathrm{cm})$ : Cintura (PC), Braço (PB), Glútea (PG), Coxa (PCX) e Panturrilha (PPM) segundo Martins e Lopes ${ }^{14}$ e dobras cutâneas: para homens (tríceps, subescapular, supra-ilíaca, panturrilha medial); para as mulheres (axilar média, supra-ilíaca, coxa e panturrilha medial) segundo Benedetti et al. ${ }^{15}$;

Resultados: com o cálculo do Índice de Massa Corporal $(\mathrm{IMC}=$ Massa $(\mathrm{kg}) /$ Estatura em metros ao quadrado), percentual de gordura por meio do somatório das dobras cutâneas ${ }^{16}$, determinação do risco pela distribuição da gordura pela medida do PC, percentual de massa magra e massa corporal magra em quilogramas, e a massa corporal esperada (mínimo e máximo) calculados; e as devidas observações e recomendações.

\section{INTERPRETAÇÃO E RECOMENDAÇÕES PARA A SUA APLICAÇÃO NO NASF}

Após realização das medidas sugeridas para a Ficha Antropométrica no NASF (Anexo 1) deve-se identificar as variáveis da composição corporal e os indicadores antropométricos para interpretação dos resultados e recomendações.

As medidas antropométricas de massa corporal (peso) e altura (estatura), o perímetro de cintura e as dobras cutâneas são as variáveis descritas neste protocolo para identificação do índice de massa corporal, do percentual de gordura e de MCM, e da distribuição da gordura corporal. Realizadas as medidas e os cálculos sugere-se uma avaliação em conjunto conforme propostas das Figuras 2 e 3.

No Quadro 1, a partir da intersecção de duas linhas imaginárias, uma classificando o PC (vertical) e a outra o IMC (horizontal), é possível determinar o perfil do risco ${ }^{17}$.

A força da relação entre as medidas aqui abordadas e os fatores de risco às principais doenças podem ser observadas na Figura 2, onde os níveis e a variação de massa corporal e os níveis e a localização da gordura determinam a força de associação para os maiores riscos à saúde cardiovascular e metabólica.

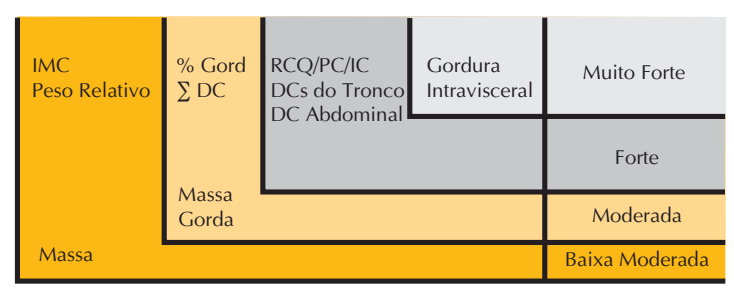

Figura 2. Relação da força das medidas e indicadores antropométricos com a predisposição a doenças e agravos a saúde. Adaptado de Abernethy et al. ${ }^{18}$. 


\begin{tabular}{|c|c|c|c|}
\hline \multirow{2}{*}{$\begin{array}{c}\text { Índice de Massa Corporal } \\
\text { (IMC) }\end{array}$} & Homens $\leq 102 \mathrm{~cm}$ & Homens $>102 \mathrm{~cm}$ \\
\cline { 3 - 4 } & & Mulheres $\leq 88 \mathrm{~cm}$ & Mulheres $>88 \mathrm{~cm}$ \\
\cline { 3 - 4 } & $<18,5$ & $\ldots$ & $\ldots$ \\
\hline Peso insuficiente & 18,5 a 24,9 & $\ldots$ & $\ldots$ \\
\hline Normal & 25,0 a 29,9 & Aumentado & Alto \\
\hline Sobrepeso & & & Muito alto \\
\hline Obesidade (classe) & 30 a 34,9 & Alto & Muito alto \\
\hline I & 35 a 39,9 & Muito alto & Extremamente alto \\
\hline II & $\geq 40,0$ & Extremamente alto & (PC) \\
\hline III & &
\end{tabular}

Quadro 1. Análise conjunta das medidas de PC e IMC para determinação de risco cardiovasculares e metabólicos (ACSM,p. 58)17.

Tabela 1. Classificação do Perímetro da Cintura (PC), segundo a $\mathrm{OMS}^{19}$.

\begin{tabular}{lcc}
\hline Classificação & Homens & Mulheres \\
\hline Aumentado & $\geq 94,0 \mathrm{~cm}$ & $\geq 80,0 \mathrm{~cm}$ \\
$\begin{array}{l}\text { Risco substancialmente } \\
\text { aumentado }\end{array}$ & $\geq 102,0 \mathrm{~cm}$ & $\geq 88,0 \mathrm{~cm}$ \\
\hline
\end{tabular}

Tabela 2. Classificação do percentual de gordura segundo de Lohman $^{20}$.

\begin{tabular}{lcc}
\hline Classificação & Homens & Mulheres \\
\hline Muito Baixo* & $\leq 5,0 \%$ & $<8,0 \%$ \\
Abaixo da Média & $6-14 \%$ & $9-22 \%$ \\
Média & $15 \%$ & $23,0 \%$ \\
Acima da Média & $16-24,0 \%$ & $24-31,0 \%$ \\
Muito Alto** & $\geq 25,0 \%$ & $>32,0 \%$ \\
\hline
\end{tabular}

* Risco para doenças e desordens associadas com a má nutrição

** Risco para doenças e desordens associadas com a obesidade.

Tabela 3. Limites desejaveis do indice de massa corporal segundo sexo e idade $\left(\right.$ Bray $\left.^{21}\right)$.

\begin{tabular}{lcc}
\hline $\begin{array}{l}\text { Grupo etário } \\
\text { (anos) }\end{array}$ & $\begin{array}{c}\text { Indice de massa corporal }\left(\mathrm{kg} / \mathrm{m}^{2}\right) \\
\text { mulheres }\end{array}$ & homens \\
\hline $19-24$ & $18-24$ & $19-24$ \\
$25-34$ & $20-25$ & $20-25$ \\
$35-44$ & $21-26$ & $20-25$ \\
$45-54$ & $22-27$ & $20-25$ \\
$55-64$ & $23-28$ & $20-25$ \\
$\geq 65$ & $24-29$ & $20-25$ \\
\hline
\end{tabular}

Desta forma, a utilização e a interpretação de um indicador para cada componente (massa corporal total, massa gorda e gordura abdominal) aumenta a determinação de risco e a eficiência de uma possível intervenção.

Dentre as várias propostas de interpretação dos resultados antropométricos para a ficha no NASF sugerese, para este modelo os seguintes critérios de análise:

a) Para o IMC e PC, os pontos de corte sugeridos pela $\mathrm{OMS}^{19}$ (Quadro 1 e Tabela 1); b) Para a determinação e a classificação do percentual de gordura as referências de Petroski ${ }^{16}$ (Figura 4 e 5) e Lohman ${ }^{20}$ (Tabela 2) respectivamente; $\mathrm{e}$

c) Para o cálculo da massa corporal esperada: os pontos de corte mínimo e máximo proposto por $\operatorname{Bray}^{21}$ (Tabela 3).

A seguir é apresentado um passo a passo para aplicação e interpretação dos dados propostos pela ficha antropométrica para o NASF:

\section{EXEMPLO}

\section{Adulto, 45 anos, sexo feminino, massa} corporal de $98 \mathrm{~kg}$, estatura de 1,76m.

\section{Medidas de Triagem}

Passo 1 - Após a verificação das medidas de massa e estatura realizar o cálculo do IMC;

$$
\begin{aligned}
& \operatorname{IMC}\left(\mathrm{kg} / \mathrm{m}^{2}\right)=\frac{\operatorname{MC}(\mathrm{kg})}{\operatorname{Est}(\mathrm{m})} \rightarrow \\
& \operatorname{IMC}\left(\mathrm{kg} / \mathrm{m}^{2}\right)=\frac{98}{(1,76)^{2}}=\frac{98}{3,09}=31,71 \mathrm{~kg} / \mathrm{m}^{2}
\end{aligned}
$$

Passo 2 - Após o cálculo ou identificação do IMC (passo 1) interpretar o valor no Quadro 1, para classificação do IMC para peso insuficiente, sobrepeso e obesidade.

$\operatorname{IMC}\left(\mathrm{kg} / \mathrm{m}^{2}\right)=31,71 \mathrm{~kg} / \mathrm{m}^{2} \rightarrow$ Obesidade Classe I - Risco alto (Tabela 2)

Passo 3 - Após a realização da medida do perímetro da cintura (PC) interpreta-se o resultado pelos seguintes valores, de acordo com o sexo (Tabela1).

$\mathrm{PC}=92,07 \mathrm{~cm} \rightarrow$ Risco Substancialmente Aumentado 
Quadro 2. Modelo Ficha Antropométrica para o Núcleo de Apoio à Saúde da Família (FANASF).

\begin{tabular}{|c|c|c|c|c|}
\hline \multicolumn{5}{|l|}{ Ficha Antropométrica } \\
\hline \multicolumn{5}{|l|}{ Identificação/ Dados Pessoais } \\
\hline \multicolumn{2}{|l|}{ Nome: Silvana da Silva } & Sexo: & ( ) Masc & ( $\mathrm{x}$ ) Fem. \\
\hline \multicolumn{2}{|l|}{ Unidade de Saúde: } & Idade: & 45 & anos \\
\hline \multicolumn{2}{|l|}{ Bairro/Endereço: } & \multicolumn{3}{|c|}{ Contato Telefônico: } \\
\hline \multicolumn{2}{|l|}{ Nome da ACS: } & \multicolumn{3}{|c|}{ No.da Micro-área: } \\
\hline Datas das medidas em Domicílio & $13 / 06 / 2008$ & & & \\
\hline Datas das medidas na UBS & $13 / 07 / 2008$ & & & \\
\hline \multicolumn{5}{|l|}{ Medidas Antropométricas } \\
\hline Medidas de Triagem & 1 & II & III & IV \\
\hline Estatura $(\mathrm{m})$ & 1,76 & & & \\
\hline Massa Corporal (kg) & 98 & & & \\
\hline Índice de Massa Corporal $\left(\mathrm{IMC} \mathrm{kg/ \textrm {m } ^ { 2 } )}\right.$ & 31,71 & & & \\
\hline Perímetro da Cintura $(\mathrm{cm})$ & 92,07 & & & \\
\hline \multicolumn{5}{|l|}{ Medidas Complementares } \\
\hline Perímetro Braço $(\mathrm{cm})$ & 30 & & & \\
\hline Perímetro Glúteo $(\mathrm{cm})$ & 100 & & & \\
\hline Perímetro Coxa $(\mathrm{cm})$ & 54 & & & \\
\hline Perímetro Panturrilha (cm) & 38 & & & \\
\hline Dobra Cutânea Subescapular (mm) - M & - & & & \\
\hline Dobra Cutânea Tricipital $(\mathrm{mm})-\mathrm{M}$ & - & & & \\
\hline Dobra Cutânea Supra-íliaca Obliqua (mm) - M e F & 27 & & & \\
\hline Dobra Cutânea Axilar Média $(\mathrm{mm})$ - F & 22 & & & \\
\hline Dobra Cutânea Coxa Media $(\mathrm{mm})-\mathrm{F}$ & 28 & & & \\
\hline Dobra Cutânea Panturrilha Medial $(\mathrm{mm})-\mathrm{M} \mathrm{e} \mathrm{F}$ & 10 & & & \\
\hline$\Sigma 4$ das Dobras Cutâneas (mm) & 87 & & & \\
\hline \multicolumn{5}{|l|}{ Resultados } \\
\hline$\%$ de Gordura & $30,17 \%$ & & & \\
\hline Massa de gordura $(\mathrm{kg})$ & $29,56 \mathrm{~kg}$ & & & \\
\hline$\%$ de Massa Corporal Magra & $69,83 \%$ & & & \\
\hline Massa Corporal Magra $(\mathrm{kg})$ & $68,44 \mathrm{~kg}$ & & & \\
\hline Classificação do IMC & Obesidade I & & & \\
\hline Classificação do perímetro da Cintura & Risco aumentado & & & \\
\hline Faixa de Massa Corporal Recomendável (min.-máx.) & 67,98 a $83,43 \mathrm{~kg}$ & & & \\
\hline Classificação do \% de Gordura & Acima da média & & & \\
\hline \multicolumn{5}{|l|}{ Recomendações } \\
\hline
\end{tabular}

$M=$ masculino $F=$ feminino

para Doenças Cardiovasculares

Passo 4 - Realiza-se a análise conjunta dos índices encontrados nos passos 2 e 3 (IMC e PC) segundo o Quadro 1, onde se observa que este indivíduo apresenta risco muito alto para a relação ao IMC associada à distribuição da gordura na região central do corpo. Logo, diante deste resultado a ACS deverá marcar o atendimento deste adulto na UBS para aferir as medidas complementares e receber orientação específica.

Com a presença do adulto na UBS seguirá a próxima etapa de aferição (medidas complementares), pelo professor de educação física, iniciando com as medidas de perímetros e em seguida das dobras cutâneas, segundo os critérios das técnicas e da padronização sugeridos no procedimento. 


\section{Medidas Complementares}

Passo 5 - Os perímetros corporais devem ser utilizados para observar as mudanças morfológicas em resposta às orientações e recomendações para adoção de hábitos saudáveis e a prática de atividade física regular.

Passo 6 - Para as dobras cutâneas, após a verificação, deve-se realizar o somatório das dobras cutâneas, que para o sexo feminino são: axilar média (DCAM), supra-ilíaca obliqua (DCSI), coxa média (DCCX) e panturrilha medial (DCPM).

$$
\begin{aligned}
& \sum 4 \mathrm{DC}=\mathrm{AM}+\mathrm{SI}+\mathrm{CX}+\mathrm{PM} \rightarrow \\
& \sum 4 \mathrm{DC}=18+27+32+10=87 \mathrm{~mm}
\end{aligned}
$$

Tendo como referência o quadro 2 e por meio do $\Sigma 4 D C$ e da idade traça-se uma linha horizontal no valor da soma das dobras cutâneas e uma linha vertical na idade (45 anos), sendo o ponto de intersecção o valor estimado do percentual de gordura corporal para mulheres ${ }^{16}$.

Para o sexo masculino utiliza-se a Quadro 3, considerando as dobras cutâneas triciptal, subescapular, supra-ilíaca e panturrilha medial.

Passo 7 - Conforme observa-se a classificação para o valor do \%GCacima indicado pela $\Sigma 4 \mathrm{DC}(\% \mathrm{G}$ =30,17) é acima da média para mulheres (Tabela 2).

Passo 8 - Identificação da massa de gordura. Massa de gordura $=$ massa total $\times \%$ de gordura $\rightarrow$ Massa de gordura $=98 \times 31,17 \%=29,56 \mathrm{~kg}$

Passo 9 - Representação da Massa Magra em percentual $(\% \mathrm{MCM})$ e quilogramas $(\mathrm{kg})$.

Massa de MCM $=$ massa total $\times \%$ de gordura $\rightarrow$ Massa de $\mathrm{MCM}=98-29,56=68,44 \mathrm{~kg}$

Passo 10 - Determinação da massa corporal esperada para saúde. Considerando o ponto de corte inferior $\left(22,0 \mathrm{~kg} / \mathrm{m}^{2}\right)$ e superior $\left(27,0 \mathrm{~kg} / \mathrm{m}^{2}\right)$ para o IMC de acordo com Bray ${ }^{21}$ (Tabela 3) e que a medida de estatura do avaliado é conhecida, mediante ajuste da equação do IMC (massa/estatura ao quadrado):

10.1 - Massa Corporal Esperada Mínima (MEmin.)

$$
\begin{aligned}
& \mathrm{IMC}_{\min }\left(\mathrm{kg} / \mathrm{m}^{2}\right)=\frac{\text { MEmin }}{\text { Estatura }^{2}} \rightarrow \\
& 22,0=\frac{\text { MEmin }}{(1,76)^{2}} \rightarrow 22,0=\frac{\text { MEmin }}{3,09} \rightarrow
\end{aligned}
$$

$$
\operatorname{MEmin}=22,0 \times 3,09=67,98 \mathrm{~kg}
$$

10.2 - Massa Corporal Esperada Máxima (MEmax.)

$$
\begin{aligned}
& \mathrm{IMC}_{\text {max }}\left(\mathrm{kg} / \mathrm{m}^{2}\right)=\frac{\text { PEmax }}{\text { Estatura }^{2}} \rightarrow \\
& 27,0=\frac{\text { PEmax }}{(1,76)^{2}} \rightarrow 27,0=\frac{\text { PEmax }}{3,09} \rightarrow
\end{aligned}
$$

$\mathrm{PEmax}=27,0 \times 3,09=83,43 \mathrm{~kg}$

Logo, diante da massa corporal esperada (entre 67,98 à 83,43 kg) observa-se a necessidade de perda da massa inicial de 14,57 kg, que conforme as medidas antropométricas e os indicadores está relacionado principalmente ao excesso de gordura corporal.

Passo 11 - Considerando os valores dos indicadores e suas respectivas classificações de risco saúde: $\mathrm{IMC}=31,71 \mathrm{~kg} / \mathrm{m}^{2}$ (obesidade $\mathrm{I}$ ), $\mathrm{PC}=$ $92,07 \mathrm{~cm}$ (risco substancialmente aumentado), \% GC=30,17(acima da média), e a faixa da massa esperada entre 67,98 à $83,43 \mathrm{~kg}$, e com outras variáveis bioquímicas e hemodinâmicas disponibilizadas nas fichas médicas, é possível direcionar uma ação multidisciplinar efetiva para mudança do quadro apresentado, minimizando os riscos predisponentes a doenças associadas e conhecidas como mais graves (acidente vascular cerebral, infarto agudo do miocárdio, isquemias, entre outras).

Passo 12 - Encaminhamento: sugere-se que estas informações fiquem anexadas e arquivadas em local apropriado na Unidade Básica de Saúde e disponível para outros profissionais, permitindo o desenvolvimento de abordagens interdisciplinares. A Figura 4 apresenta a organização das medidas com suas respectivas etapas e procedimentos da ficha antropométrica para o NASF.

\section{CONSIDERAÇÕES FINAIS}

A participação de outros profissionais da área da saúde prevista com a criação dos Núcleos de Apoio a Saúde da Família passa a ser uma importante iniciativa para a saúde pública. Porém, deve-se considerar que com esta nova perspectiva de atuação exista a necessidade de novos conhecimentos e procedimentos para efetivar as ações e contribuições destes profissionais.

A Ficha Antropométrica no NASF deve ser reconhecida inicialmente como uma proposta que oriente as ações do professor de Educação Física para 
Quadro 2. Percentual de gordura corporal para mulheres acima de 18 anos de idade a partir da soma das dobras cutâneas axilar média, supra-ilíaca obliqua, coxa medial e panturrilha medial (Petroski, 1995) ${ }^{16}$.

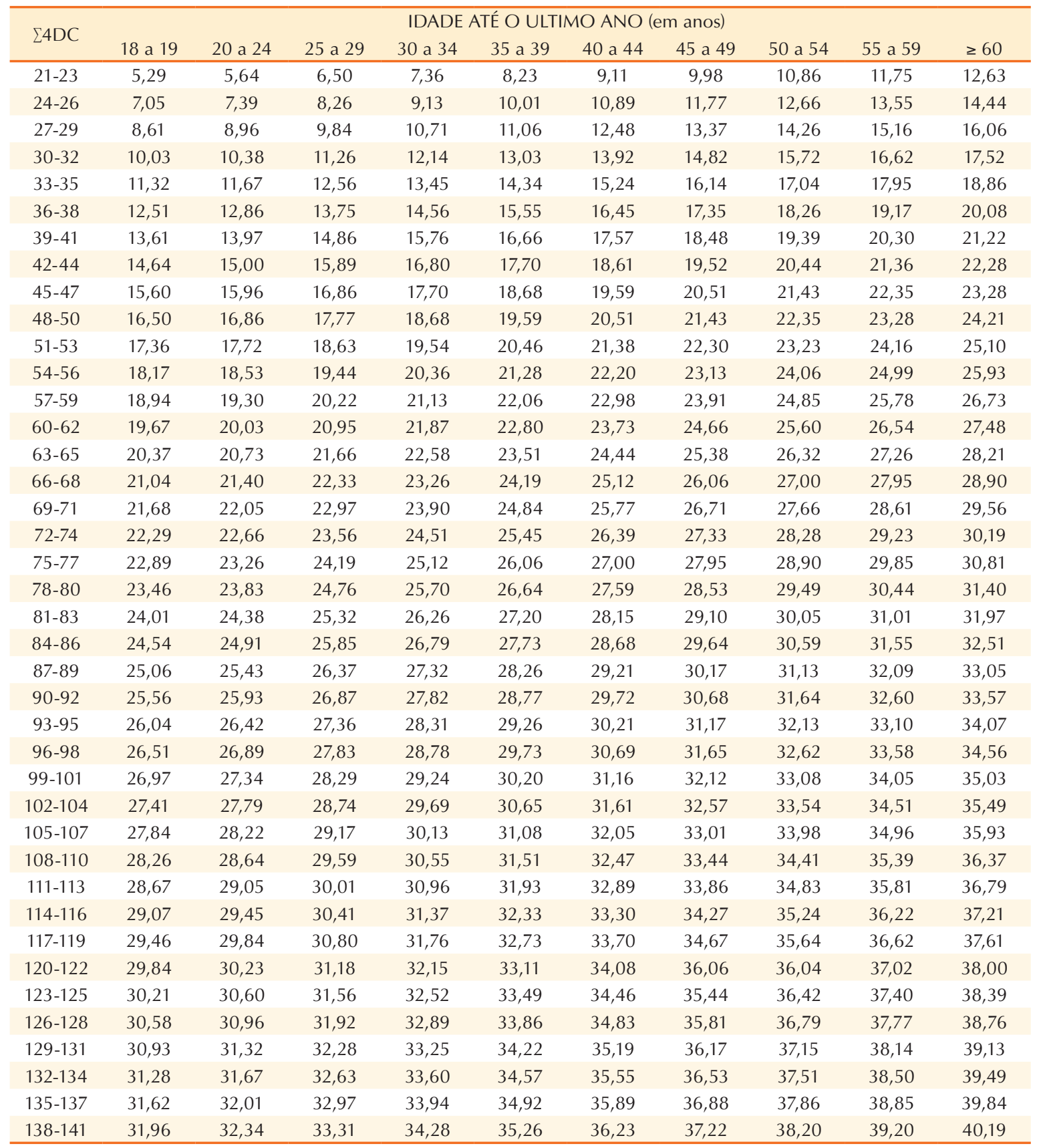

a promoção da saúde, pois na sua construção e planejamento foram contemplados aspectos técnicos e logísticos, baseados em estratégias, onde a prevenção e as práticas de educação à saúde são recomendadas.

Reforçando a necessidade da prática da promoção à saúde, a utilização da Ficha Antropométrica no NASF deve fazer parte de uma intervenção mais abrangente e específica para a atuação do professor de Educação Física, como observado no estudo de Gomes e Duarte ${ }^{22}$, que apresenta uma proposta de ação para orientação de atividade física na ESF.
Outro ponto a se destacar para a atuação da Educação Física e o uso da antropometria no NASF, é a instrumentalização dos professores de Educação Física que atuam nas escolas pertencentes às áreas de cobertura da ESF, estimulando-os a utilização da Ficha Antropométrica Escolar ${ }^{23}$, e favorecendo a intersetorialidade entre a Educação e a Saúde.

A permanente comunicação e troca de experiências e conhecimentos entre os integrantes da equipe (intervenção multiprofissional), juntamente com o saber popular das ACS constitui-se elemen- 
Quadro 3. Percentual de gordura corporal para homens acima de 18 anos de idade a partir da soma das dobras cutâneas triciptal, subescapular, supra-ilíaca e panturrilha medial (Petroski, 1995) ${ }^{16}$.

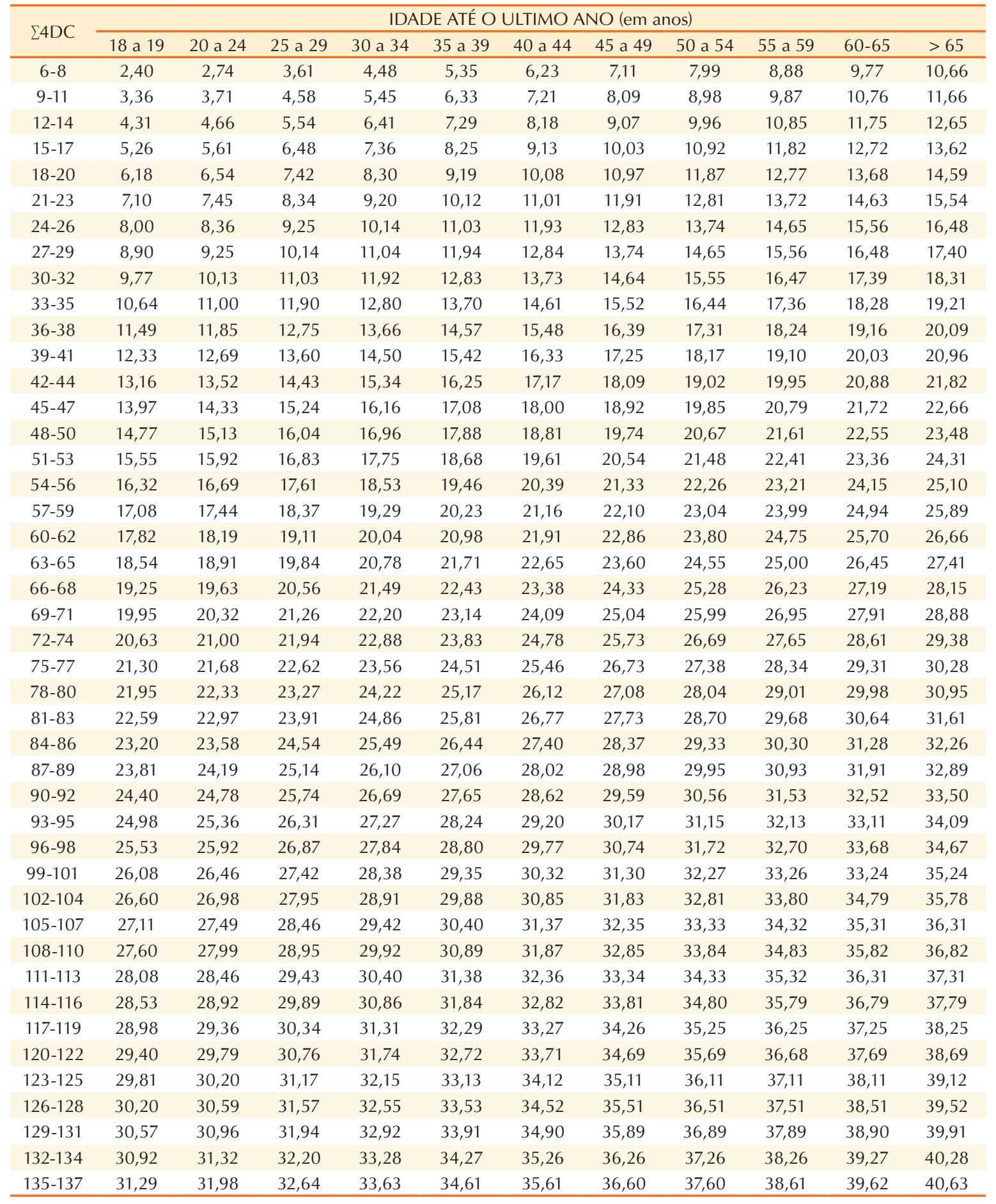

to-chave para efetivação das ações da promoção da saúde sugeridas pela ficha.

Para evitar a diminuição/limitação da abrangência na ação do professor de educação física, deve-se estar atento para que esta proposta de atuação na saúde pública não seja vista apenas no contexto clínico/consultório e sim como uma possibilidade de reforçar/ampliar as iniciativas educacionais no campo da promoção a saúde.
Por fim, a ação do professor de educação física e a aplicação da antropometria no NASF, além de proporcionar a detecção de fatores de risco (prevenção), deve evidenciar as estratégias de educação à saúde, possibilitando assim, que os profissionais envolvidos possam mediar às ações de promoção a saúde por meio de prática efetiva, transformadora, autônoma e crítica da realidade. 


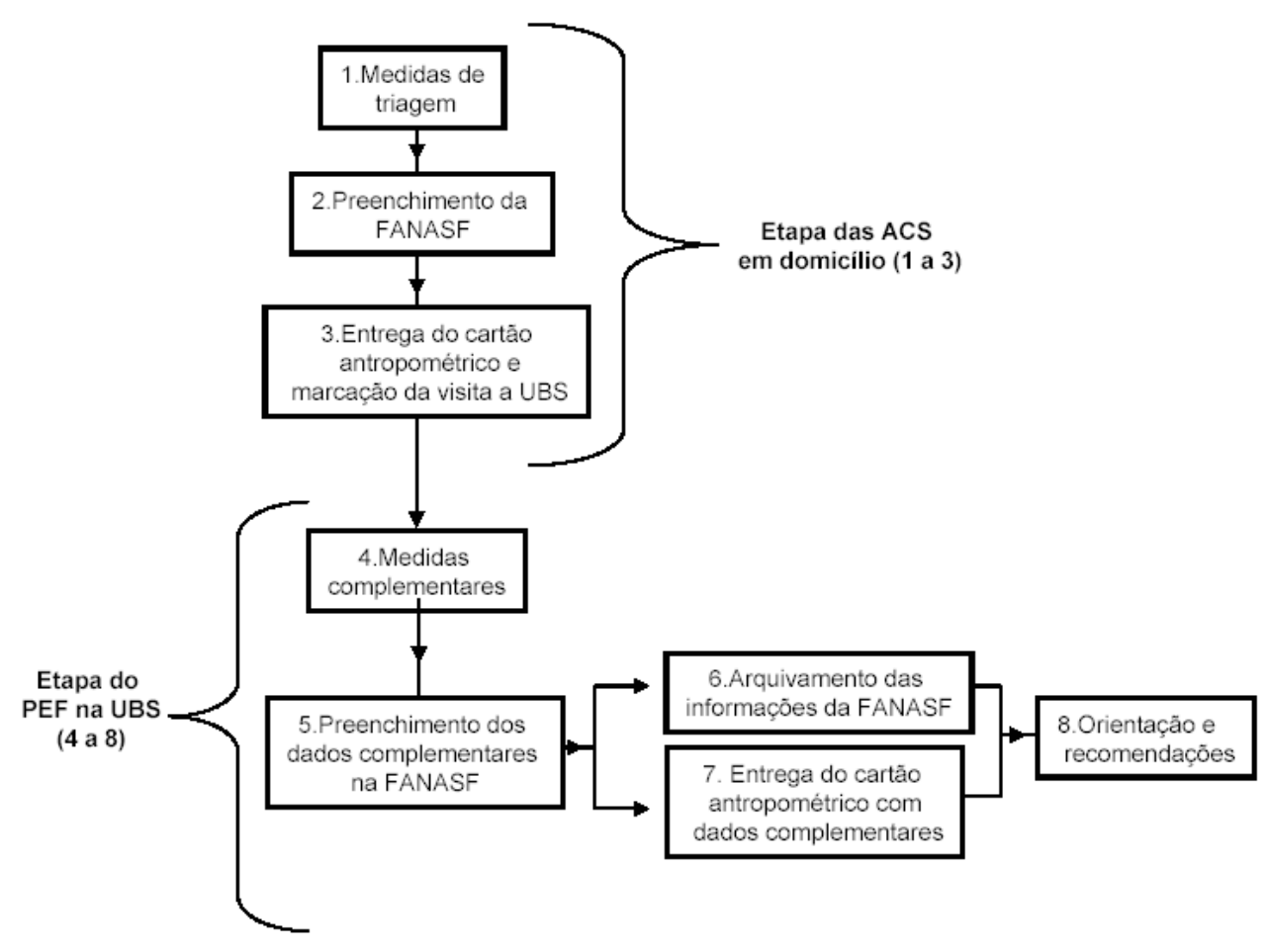

ACS: Agente Comunitário de Saúde, Ficha antropométrica do NASF: Ficha Antropométrica No Núcleo de Apoio a Saúde da Família, PEF: Professor de Educação Física, UBS: Unidade Básica de Saúde.

Figura 3. Etapas e procedimentos das medidas da ficha antropométrica do NASF.

\section{REFERÊNCIAS BIBLIOGRÁFICAS}

1. Organização Pan-Americana de Saúde. Carta de Ottawa: primeira conferência internacional sobre promoção da saúde. 2006 [Acesso em 2008 Out 12] Disponível em: http://www.opas.org.br/coletiva.

2. Brasil, Ministério da Saúde. Projeto promoção da saúde. As cartas da promoção da saúde; Secretaria de Políticas de Saúde; Brasília, Governo Federal: Ministério da Saúde; 2002.

3. Brasil, Ministério da Saúde. Política nacional de promoção da saúde (Documento para discussão). Brasília. 2002; 42.

4. Brasil, Ministério da Saúde. Saúde da Família: uma estratégia para a reorientação do modelo assistencial. Brasília: Governo Federal; Ministério da Saúde; 1994.

5. Brasil, Ministério da Saúde. Sistema de Gestão Federal/ Estadual de Vigilância Alimentar e Nutricional (SISVAN). 1990. [acesso em 2008 mai 02]. Disponível em: http://sisvan.datasus.gov.br/index.asp

6. Núcleo de Apoio da Saúde da Família. Portaria Ministerial de Saúde nº 154, de 24 de janeiro de 2008. [acesso em 2008 Abr 30]. Disponível em: http://www. saude.sc.gov.br/PSF/PORTARIAS/PORTARIA\%20 GM\%20N154.pdf

7. Brasil, Ministério da Saúde, MS/ Departamento de Atenção Básica. Atenção básica e saúde da família. 2008 [acesso em 2008 mai 02]. Disponível em: http:/ dtr2004.saude.gov.br/dab/atencaobasica.php\#equipes
8. Brasil, Ministério da Saúde. Saúde da Família: uma estratégia para a reorientação do modelo assistencial. Brasília: Governo Federal; Ministério da Saúde; 1994.

9. Brasil, Ministério da Saúde (M S). Avaliação da implantação e funcionamento do Programa de Saúde da Família. Brasília, Governo Federal: Ministério da Saúde. 2000.

10. Gomes MA. Orientação de atividade física em programa de saúde da família: uma proposta de ação. [Dissertação de Mestrado - Programa de Pós-Graduação em Educação Física]. Florianópolis (SC): Universidade Federal de Santa Catarina; 2007.

11. Freire P. Pedagogia da autonomia: saberes necessários para a prática docente. São Paulo: Paz \& Terra; 1996.

12. Buss PM. Uma introdução ao conceito de promoção da saúde. In: Czeresnia D, Freitas CM. Promoção da Saúde: conceitos, reflexões e tendências. Rio de Janeiro: FIOCRUZ; 2003. p. 9-14.

13. Alvarez BR, Pavan AL. Alturas e Comprimentos. In: Petroski EL, editor. Antropometria: técnicas e mensurações. 4 ed. Porto Alegre: Editora Pallotti; 2009. p. 31-44.

14. Martins MO, Lopes MA (2009). Perímetros. In: Petroski EL, editor. Antropometria: Técnicas e Mensurações. 4 ed. Porto Alegre: Editora Pallotti; 2009. p.57-69.

15. Benedetti TRB, Pinho RA, Ramos VM. Dobras Cutâneas In: Petroski EL, editor. Antropometria: técnicas e mensurações. 4 ed. Porto Alegre: Editora Pallotti; 2009. p. 45-56.

16. Petroski EL. Equações Antropométricas: subsídios para uso no estudo da composição corporal. In: Petroski EL, 
editor. Antropometria: técnicas e mensurações. 4 ed. Porto Alegre: Editora Pallotti; 2009. p.121-139.

17. ACSM. Diretrizes do ACSM para os testes de esforço e sua prescrição. Rio de Janeiro: Guanabara Koogan, 2007. p.45.

18. Abernethy P, Olds T, Éden B, Neill M, Baines L. Antropometria, saúde e composição corporal. In: Norton K, Olds T, editores. Antropométrica. Porto Alegre: Artmed; 2005. p. 347-371.

19. Organização Mundial de Saúde. Obesidade: prevenindo e controlando a epidemia global. São Paulo: Editora Roca; 2004.

20. Lohman TG. Advances in body composition assessment. In: Human Kinetics Champaign,1992.

21. Bray GA.'Overweight is risking fate, definition, classification, prevalence and risk". NYAS.1987;249:14-28.

22. Gomes MA, Duarte MFS. Efetividade de uma intervenção de atividade física em adultos atendidos pela estratégia saúde da família: Programa Ação e Saúde Floripa- BRASIL. RBAFS. 2008;13(1):44-56.

23. Beck CC, Diniz IMS, Gomes MA, Petroski EL. Ficha antropométrica na escola: o que medir e para que medir? Rev Bras Cine Des Hum. 2007;9(1):107-114.

\section{Endereço para correspondência}

Marcius de Almeida Gomes

Universidade do Estado da Bahia

Departamento de Educação - Campus XII

Avenida Universitária Vanessa Cardoso e Cardoso, Loteamento Ipanema, Bairro Ipanema, s/n, Campus Universitário. Guanambi, BA. Brasil, CEP:46430-000.

E-mail: magomes@uneb.br 